\title{
The EMIR multi-band mm-wave receiver for the IRAM 30-m telescope ${ }^{\star}$
}

\author{
M. Carter ${ }^{1}$, B. Lazareff ${ }^{1,2}$, D. Maier ${ }^{1}$, J.-Y. Chenu ${ }^{1}$, A.-L. Fontana ${ }^{1}$, Y. Bortolotti ${ }^{1}$, C. Boucher ${ }^{1}$, A. Navarrini ${ }^{1}$, \\ S. Blanchet ${ }^{1}$, A. Greve ${ }^{1}$, D. John ${ }^{3}$, C. Kramer ${ }^{3}$, F. Morel ${ }^{1}$, S. Navarro ${ }^{3}$, J. Peñalver ${ }^{3}$, K. F. Schuster ${ }^{1}$, and C. Thum ${ }^{1,3}$ \\ ${ }^{1}$ Institut de Radioastronomie Millimétrique, 300 rue de la Piscine, 38406 St Martin d'Hères, France ${ }^{\star \star}$ \\ 2 Insitut de Planétologie et d'Astrophysique de Grenoble, BP 53, 38041 Grenoble Cédex 9, France \\ e-mail: bernard.lazareff@obs.ujf-grenoble.fr

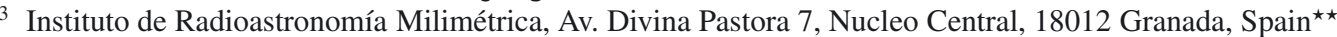

Received 14 November 2011 / Accepted 30 November 2011

\begin{abstract}
Aims. The prime motivation of this project was to design and build a state-of-art mm-wave heterodyne receiver system to enhance the observing throughput of the IRAM 30-m radiotelescope. More specifically, the requirements were i) state-of-art noise performance for spectroscopic observations; ii) simultaneous dual polarization and dual-frequency observing; iii) coverage of the atmospheric transmission windows from 83 to $360 \mathrm{GHz}$; iv) compact footprint and minimal maintenance.

Methods. Key elements for low noise performance of heterodyne mixers are the superconducting Niobium junctions, operating at $\simeq 4 \mathrm{~K}$. These junctions are embedded in carefully designed coupling structures; furthermore, since atmospheric radiation is a significant contributor to the system noise budget, all mixers are either sideband separating or sideband rejecting. To achieve low noise, it is also essential to maximize the coupling of the receiver to the astronomical source, and to minimize the coupling to thermal radiation from the ground-based environment; this is achieved through mirror optics that realize a wavelength-independent coupling to the telescope. A flexible configuration of mirrors and frequency selective surfaces permits various combinations of frequency bands, as well as dual-load radiometric calibration. Low noise intermediate frequency amplifiers and bias electronics also play an important role in the system performance.

Results. The EMIR receiver in operation at the $30 \mathrm{~m}$ telescope offers four frequency bands: B1: 83-117 GHz, B2: 129-174 GHz, B3: 200-267 GHz, and B4: 260-360 GHz. In each band, the two orthogonal polarizations are observed simultaneously. Dual-band combinations B1/2 B1/3, and B2/4 are available. Bands 1 and 4 (also 3 as of Nov.-2011) feature sideband separation. In dual-band configuration, including sideband separation and polarization diplexing, up to eight IF channels are delivered to the spectrometers, totaling up to $64 \mathrm{GHz}$ of signal bandwidth (of which $32 \mathrm{GHz}$ can be transported and processed by spectrometers, status Nov.2011). The EMIR receiver has been in continuous operation for more than two years and has allowed, through a qualitative jump in performance, observations not possible before, as shown by a few selected examples of astronomical results.
\end{abstract}

Key words. instrumentation: detectors - techniques: miscellaneous

\section{Introduction}

The mm-wave spectral range is well matched to the investigation of the cold interstellar matter, especially but not only in molecular form. Large single dish radiotelescopes offer, compared with interferometers, a large flexibility both in terms of observing strategy and in re-configuring the focal plane instrumentation, either for visitor instruments, or for long term upgrades. Due to the large capital investment in the reflecting telescope, improvements of the receiving electronics - whether front end or spectrometers - provide an attractive upgrade path for the instrument. In 2006, a new generation of receivers for the IRAM Plateau de Bure interferometer was in the construction phase. It was decided to capitalize on that design and derive from it a similar front end for the 30-m telescope, with enhanced features. The top-level motivations behind that decision were a) to improve the scientific throughput of heterodyne mm-band observations; b) to use the opportunity to pack a multi-band instrument into

$\star$ This article is dedicated to the memory of our colleague Matt who initiated and played a key role in this project.

$\star \star$ IRAM is co-funded by the following agencies: CNRS (France), MPG (Germany) and IGN (Spain). a relatively small volume, and make space available in the $30 \mathrm{~m}$ receiver cabin for other future instruments: bolometers or heterodyne multibeam cameras.

The improvement of the scientific throughput relies on a number of key performance specifications: i) receiver noise performance; ii) sideband rejection, that allows the rejection of (most of) the atmospheric noise and confusing astronomical lines from the image band; iii) sideband separation, making both sidebands available simultaneously; iv) wide instantaneous bandwidth; v) simultaneous dual polarization observing, that improves the $\mathrm{S} / \mathrm{N}$ and gives a polarimetric capability; vi) simultaneous dual-band observing.

The new multi-band receiver was commissioned in March 2009, and was named EMIR, for Eight MIxer Receiver.

The article is organized as follows. In Sect. 2, we give the top level requirements for the system, and a breakdown into subsystems and their functions. In the following sections, we return to each subsystem to describe in more detail its realization. Then, we report on the as-built performance. Finally, illustrative scientific results are presented.

We have tried to write this article for an audience broader than just mm-wave instrumentalists, giving explicit definitions 
Table 1. RF and IF configurations and bandwidths, status as installed in March 2009.

\begin{tabular}{lccc}
\hline \hline Band\# & $\begin{array}{c}\text { RF coverage } \\
(\mathrm{GHz})\end{array}$ & Mixing scheme & $\begin{array}{c}\text { IF config. } \\
\text { Pol } \times \text { Sb } \times \text { BW }(\mathrm{GHz})\end{array}$ \\
\hline B1 & $83-117$ & 2SB & $2 \times 2 \times 8$ \\
B2 & $129-174$ & SSB & $2 \times 1 \times 4$ \\
B3 & $200-267$ & SSB & $2 \times 1 \times 4^{a}$ \\
B4 & $260-360$ & 2SB & $2 \times 2 \times 4^{b}$ \\
\hline
\end{tabular}

Notes. The band designations will be used through the rest of the article. The following upgrades to the mixing and/or IF capability are scheduled for Nov. 2011: ${ }^{(a)}$ Band 3: 2SB mixers, $2 \times 2 \times 8 \mathrm{GHz}^{(b)}$ Band 4: $2 \times$ $2 \times 8 \mathrm{GHz}$.

and an occasional elementary equation; however, we could obviously do this only in a limited extent. Interested readers are referred to a textbook such as Tools of Radioastronomy (Wilson et al. 2009).

The IRAM staff strives to keep improving the EMIR system and the 30-m telescope; accordingly, potential observers are invited to obtain up-to-date information on the current status on IRAM's web site.

\section{System overview}

For the purpose of this overview, the EMIR receiver can be broken down into a number of subsystems. Please refer to Fig. 1.

\subsection{System requirements}

At the outset of the project, the system requirements were defined as follows.

- Four frequency bands, with RF coverage as shown in Table 1.

- Mixing scheme:

- Either SSB (image frequency rejected), with a choice of either the upper or lower sideband being the signal band.

- Or 2SB (two sideband) with both the upper and lower RF sidebands converted to separate IF outputs.

- IF bandwidth, per IF channel: $4 \mathrm{GHz}$ minimum; $8 \mathrm{GHz}$ goal.

- Dual polarization.

- Capability to observe two frequency bands simultaneously (each dual polarization), with at least the B1/B3 and B2/B4 combinations available; retain capability to observe without frequency multiplexing to minimize optical losses.

- Full remote control of all functions.

- No cryogenic fluid refills: closed cycle cryocooler.

\subsection{Optics}

The interfaces of the optics subsystem are: at the input, the $f / D=9.7$ Nasmyth focus of the $30 \mathrm{~m}$ telescope; at the output, the waveguide flange between each conical horn and the corresponding mixer input. There are actually two inputs (A/B), at two points in the telescope's focal plane; switching between these inputs is simply a matter of changing the telescope pointing offsets. There are eight outputs - four frequency bands (B1/B2/B3/B4), dual polarization (H/V) -, one for each mixer, whence the name of the system.

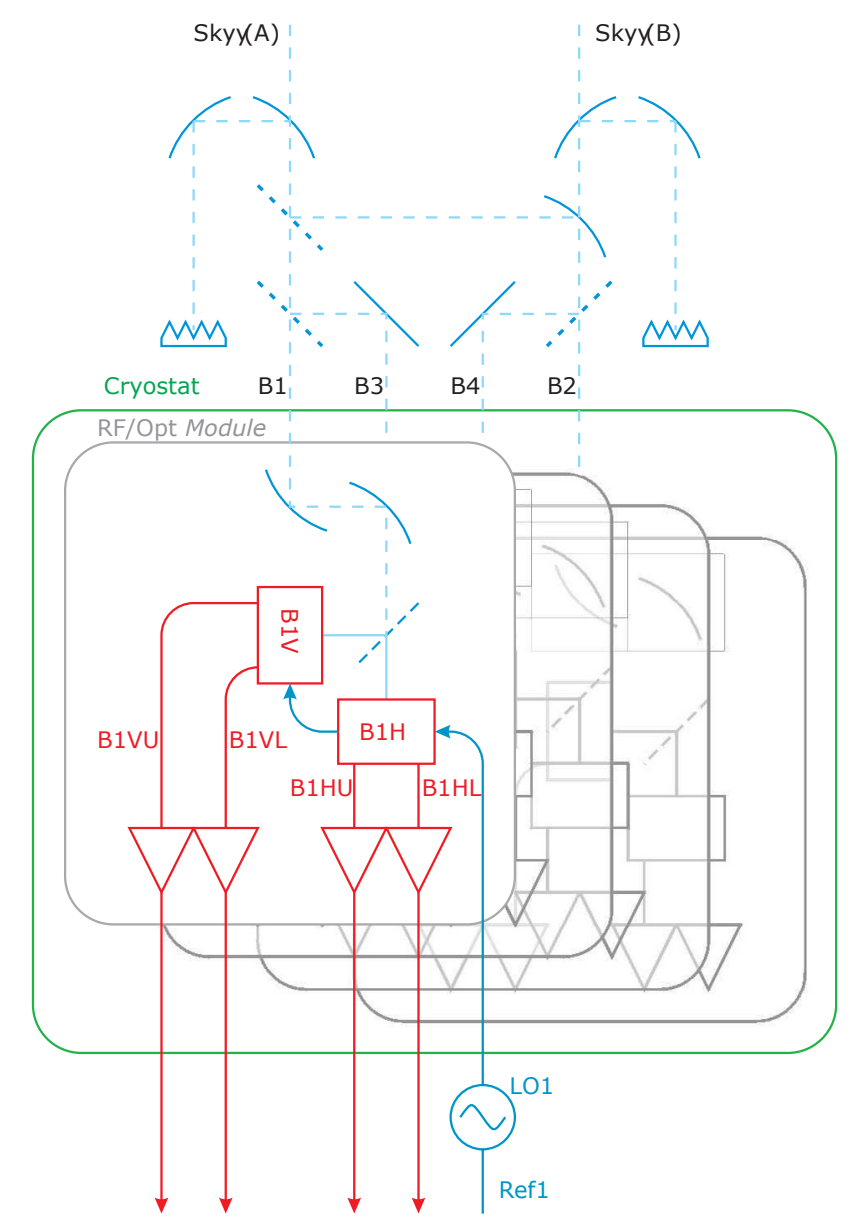

Fig. 1. Functional system diagram of the EMIR instrument. Optics: blue; Signal electronics: red; Cryogenics: green; Local oscillator: blue. Besides, the perimeter of the RF/Opt module, a building unit, is also shown. Several components are not shown. The configuration of mirrors as shown is illustrative but not realistic, a specific description can be found in Sect. 3.

Between these interfaces, the optics subsystem realizes several functions:

- match the telescope exit pupil (located at the subreflector) to the mouth of the corrugated horn, and match the latter to its output waveguide flange;

- steer the signal from one of the inputs to one pair (single frequency mode) or two pairs (dual frequency mode) of mixer inputs;

- steer each of the two linear polarizations to an individual mixer;

- perform a calibration of system gain and noise by the twoload method.

The optics subsystem is physically split in two parts: room temperature, and cold optics, the latter being located inside the cryostat.

\subsection{Mixers and signal electronics}

The signal electronics subsystem receives the astronomical signals at the waveguide flange of the horns, in one or more of the four bands, and performs the following functions:

- heterodyne down-conversion of a frequency slice of the RF (mm-wave) signal to the IF (microwave band, $4-8 \mathrm{GHz}$ or $4-12 \mathrm{GHz}$ ), with a minimum of added noise; 
- rejection of one of the $\mathrm{RF}$ side bands, or separation of the side bands, meaning the each of the two IF outputs contains the mixing product from only one of the RF side bands;

- amplification of the IF signal up to a level significantly above the noise of room temperature electronics.

A key component of each mixer is one or several superconducting tunnel junctions.

\subsection{Local oscillator}

That local oscillator (LO) subsystem provides a monochromatic signal to each mixer, allowing the heterodyne down-conversion.

\subsection{Cryogenics}

Because the superconducting tunnel junctions reach optimum performance when cooled down to $T \simeq 4 \mathrm{~K}$, and likewise the first stages of low noise IF amplification require $T \lesssim 15 \mathrm{~K}$, a cryogenic system is needed to cool these critical components.

\section{Optics}

\subsection{Frequency diplexing}

Frequency diplexing relies on dichroic mirrors, also known as frequency selective surfaces (FSS). They were commercially procured (QMC Instruments 2007), and act as low pass filters: a lower-frequency band is transmitted, while a higherfrequency band is reflected. Because a "brickwall" type of transmission versus frequency curve is not achievable, diplexing between non-adjacent bands, like B1/B3 or B2/B4 is favoured, although a compromise was made for the scientifically desirable pair B1/B2.

At room temperature, close to the cryostat top, three dichroic filters and a flat solid mirror are mounted on a translation stage; see Fig. 6. Each of those four components has a reflection angle of $45^{\circ}$ (incidence angle $22.5^{\circ}$ ). In their working configuration, they are placed above the $\mathrm{B} 1$ or $\mathrm{B} 2$ cryostat window. Furthermore, flat mirrors R3/4 above the cryostat's signal windows for B3 and B4 cause the corresponding receiver channels to permanently stare at the point above, respectively, the B1 and B2 signal window, where one of the abovementioned dichroic filters or mirrors can be inserted. Finally, two carousels located further out (from the receiver) than the dichroic mirrors, above the B1 and B2 signal windows, carry a number of elements, mostly for calibration, but also an elliptical mirror that can be located above the $\mathrm{B} 2$ window and let it receive the beam reflected from above the $\mathrm{B} 1$ window.

Rather than describe in words every possible combination, we refer the reader to Table 2 and Fig. 2.

The performance goals for the dichroic filters were a challenging combination of broad band $\left(f_{2} \div f_{1}=1.35 \ldots 1.40\right)$ and low loss $(\ell \leq 0.03)$, for both the transmitted (lower) frequency band and the reflected (higher) frequency band. The dichroic filters were characterized at component level before integration. The loss is measured using a low noise receiver staring at a load. Three measurements are made in sequence, and the IF power $P$ at the output of the receiver is measured in each configuration.

- Staring at room temperature load: $P_{\mathrm{h}}$.

- Staring at cold (LN2) load: $P_{\mathrm{c}}$.

- Staring at cold (LN2) load, in transmission or reflection through the device under test: $P_{\text {cdut }}$.
Table 2. Dichroic filters and mirror combinations used in various observing modes.

\begin{tabular}{lccc}
\hline \hline Band(s) & Filt & Car\#2 & Sky position \\
\hline B1 & & & A \\
B2 & & B \\
B3 & MR34 + R3 & & A \\
B4 & MR34 + R4 & B \\
B1+B3 & D13 + R3 & & A \\
B2+B4 & D24 + R4 & & B \\
B1+B2 & D12 & MR21 & A \\
\hline
\end{tabular}

Notes. Abbreviations as follows: filt: filter or mirror position used on translation stage; Car\# 2: elements of that carousel in active position. Mirrors R3 and R4 are fixed, but are mentioned insofar as they play a role in the signal path. Also refer to Fig. 2.

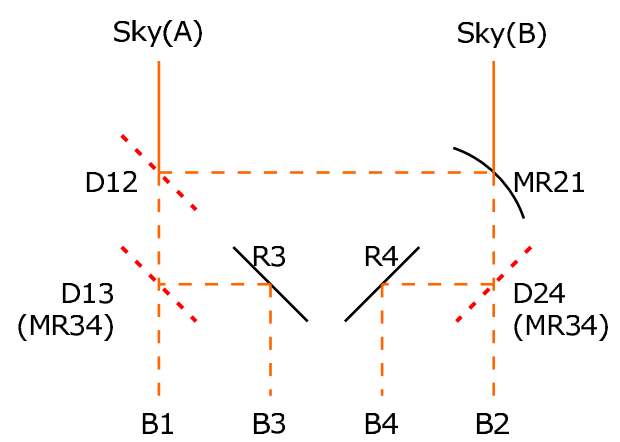

Fig. 2. Frequency diplexing functions. Top and bottom: the two sky ports (actually in the Nasmyth focal plane) and the cryostat signal windows, one per band. Dnn: dichroic mirror, with reference to band numbers. MR21: solid refocusing mirror used only in conjunction with D12. R3/4: solid mirrors in a permanent position. See also Table 2. All elements are represented in their working position, which does not correspond to a real situation. The four cryostat windows B1-4 are represented in a plane, while actually they are a the corners of a square $130 \mathrm{~mm}$ on a side. Example 1: frequency diplexing of B1/2: D12 is used jointly with MR21; D13 and D24 are outside the beams. Example 2: B3 alone (minimum loss) a solid mirror replaces D13; R3 is a fixed mirror; all other mirrors are outside the beam.

The fractional power loss is derived as:

$\ell=\frac{P_{\mathrm{cdut}}-P_{\mathrm{c}}}{P_{\mathrm{h}}-P_{\mathrm{c}}}$

That result is independent of the cold load temperature, and of the geometrical coupling of the receiver to the cold load, as long as they are the same for the second and third measurements.

The results for the three dichroic filters share a common pattern. The transmission losses, with some scatter, generally meet (or are close to meeting) the goal $\ell \leq 0.03$; besides, they are independent of the position angle (azimuth) of the device. See, e.g., Fig. 3. The reflection losses, however, show at certain frequencies a four-fold $(\cos (4 \theta))$ azimuth dependence. See, e.g., Fig. 4. Following these measurements, the dichroic filters were positioned at a "best compromise" position angle.

\subsection{Calibration}

For each of the seven observing configurations listed in Table 2, it is possible to couple the one or two receiver(s) in operation to either an ambient temperature load or a cold load; see Fig. 5. The two cold loads CL1/2 are located inside the cryostat, tied to the $15 \mathrm{~K}$ stage, and coupled through a low-loss high density 


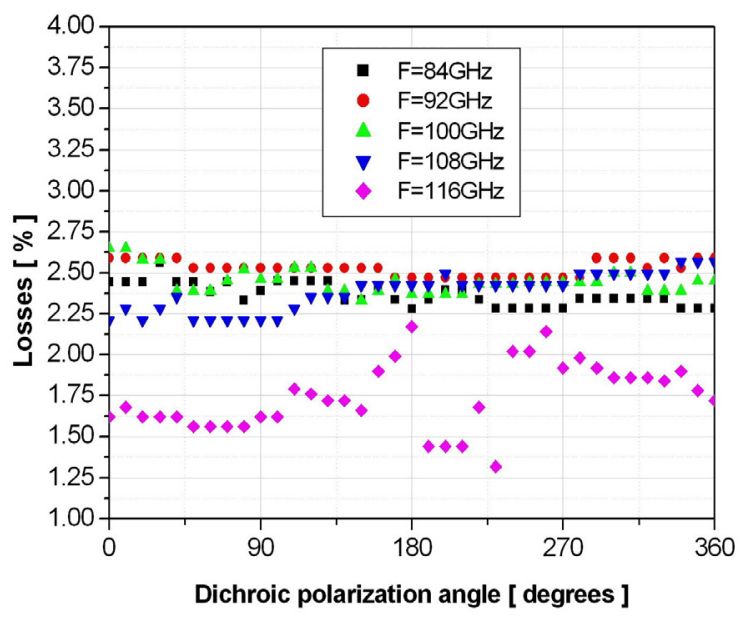

Fig. 3. Transmission loss of the B1/B3 dichroic mirror, measured in transmission at various Band1 frequencies, plotted versus the azimuth of the device.

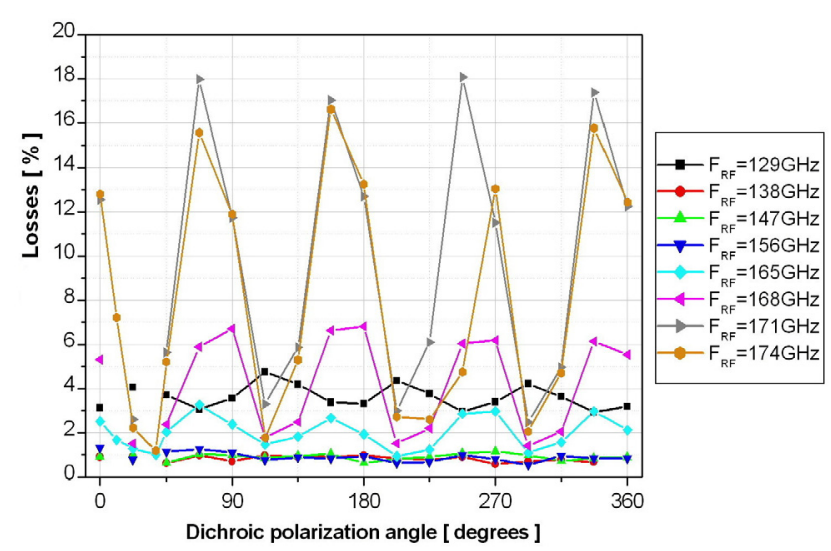

Fig. 4. Transmission loss of the B1/B2 dichroic mirror, measured in reflection at various Band2 frequencies, plotted versus the azimuth of the device.

polyethylene (HDPE) window by the pairs of refocusing mirrors $\mathrm{MC} 1 / 2$, located on their respective carousels. The two ambient temperature loads AL1/2 are also located on the corresponding carousels.

For ambient temperature calibration, a load AL1 or AL2 is inserted, via a suitable rotation of either carousel, in the beam of receiver channel B1 or B2. For cold load calibration, again by a suitable rotation of the appropriate carousel, the pair of elliptical mirrors $\mathrm{MC} 1$ or $\mathrm{MC} 2$ couples the active receiver(s) to the internal cold load(s). Because of the imperfect thermal coupling of the cold loads to the cryogenic stage, and the small but still significant losses (MC1/MC2 spillover, cryostat calibration window) the internal cold load is actually a surrogate (secondary standard) for a Nitrogen cold load located outside the cryostat; the cross-calibration is performed in the laboratory at a series of frequencies. As an illustrative example, a 3D view of the configuration for calibration in a dual-band mode is shown in Fig. 6.

The calibrated (excluding telescope coupling and atmosphere) intensity for narrow-band (i.e. appearing in only one sideband) astronomical signals is derived from:

$T_{\mathrm{SSB}}=P_{\mathrm{IF}} \times \frac{T_{\mathrm{H}}-T_{\mathrm{C}}}{P_{\mathrm{H}}-P_{\mathrm{C}}} \times \frac{1}{1+G_{\mathrm{Im}}}$

A89, page 4 of 13

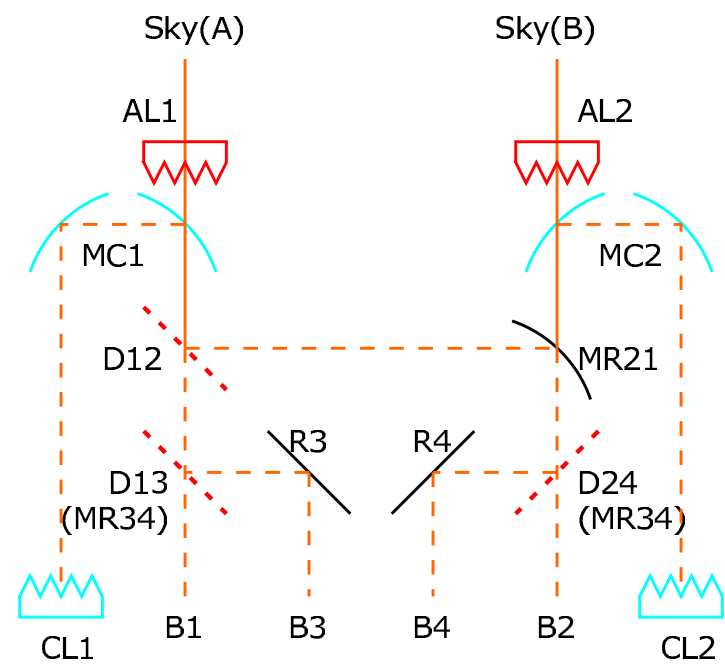

Fig. 5. Calibration functions. All elements are represented in their working position, which does not correspond to a real situation. See text.

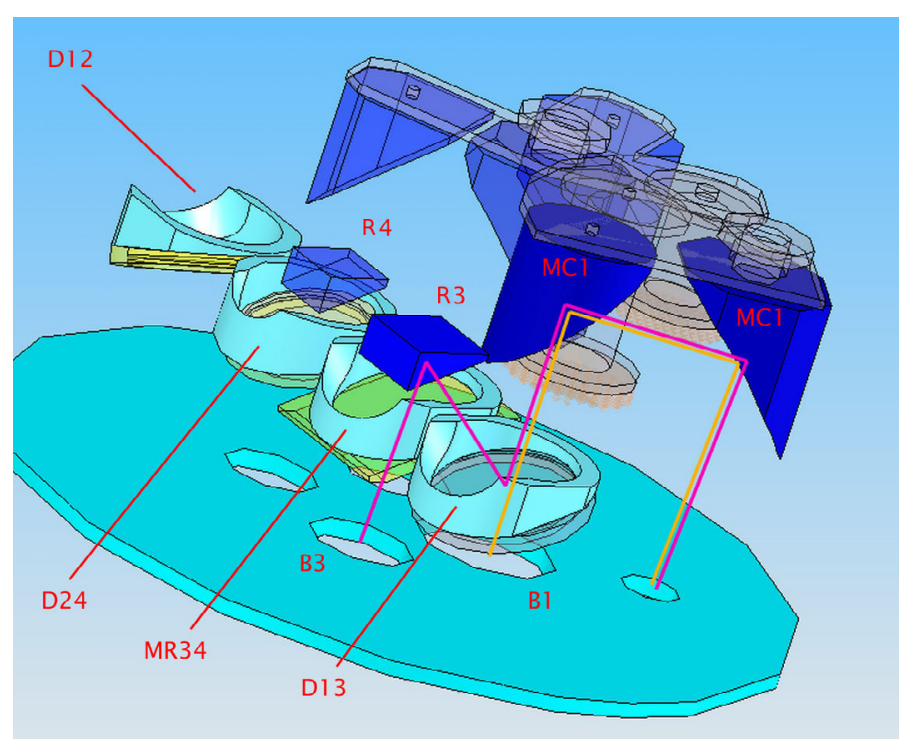

Fig. 6. A 3D rendering of the warm optics configuration for cold load calibration in dual-band B1/B3 mode. Several optical components are identified using the same nomenclature as in Figs. 5 and 2.

with $T_{\mathrm{SSB}}$ the intensity on the Rayleigh-Jeans scale, referred to the receiver input, $P_{\mathrm{IF}}, P_{\mathrm{H}}, P_{\mathrm{C}}$ the output power measured for the signal, ambient load, and cold load respectively, $T_{\mathrm{H}}$ and $T_{\mathrm{C}}$ the assigned effective temperatures of the calibration loads, and $G_{\operatorname{Im}}$ the gain in the image band relative to the signal band. Measurements of the raw IF signal show that a small amplitude standing wave pattern is present in the calibration configurations (ambient or cold load). The worst case is B1 ambient calibration, where those standing waves may induce, with respect to the broad-band cross-calibration against the LN2 load, a $2.5 \%$ maximum error in the calibration scale. Another potential source of calibration error is the image band gain. Anticipating the results shown in Figs. 12 and $16, G_{\mathrm{Im}}$ shows significant scatter on small scales of the frequency (RF or IF), but this is essentially an artifact of the logarithmic scale. In fact $G_{\mathrm{Im}}$ is generally below $0.1(<-10 \mathrm{~dB})$; accordingly, if one adopts a generic value of $G_{\mathrm{Im}}=0.05$, the worst case error induced on the calibration is $5 \%$. In many cases, especially near the center of the IF 


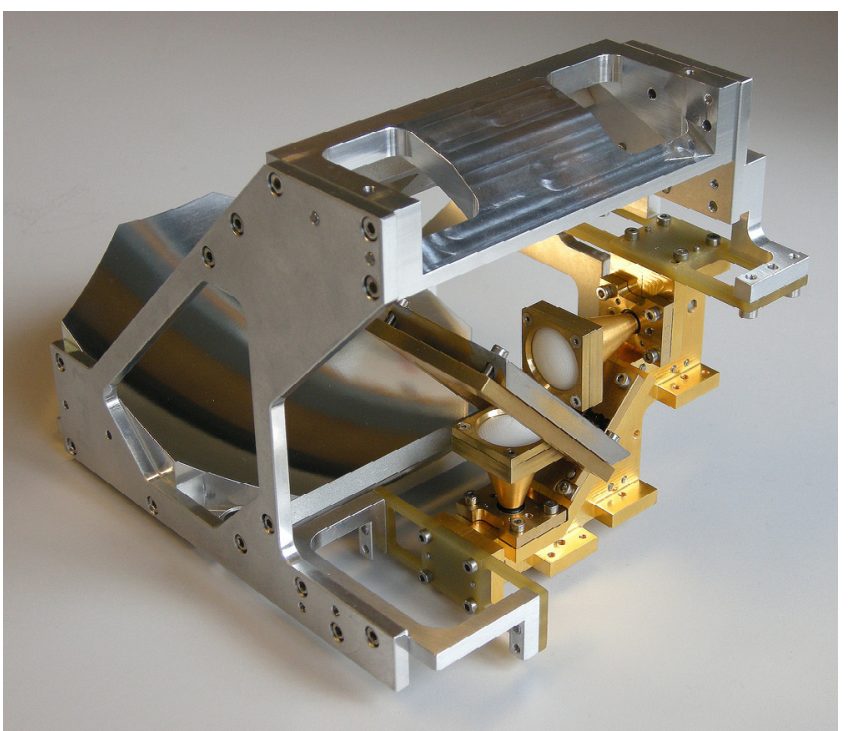

Fig. 7. The Band 3 cold optics. In the foreground, the two horns located symmetrically on either side of the polarization diplexing wire grid (seen edge on). Behind each horn sits one local oscillator injection coupler, and between the two couplers, diagonally, the LO power splitter that also serves as a mechanical support for the other elements. That sub-assembly, which is cooled to $4 \mathrm{~K}$, is mechanically linked to, and thermally insulated from the mirror sub-assembly by fiberglass-epoxy (G11) supports.

bandpass, a more stringent upper limit can be used, with correspondingly smaller calibration uncertainty.

\subsection{Coupling to telescope}

The optics subsystem of the receiver must match the input port of the electronics subsystem - single-mode rectangular waveguide - to the aperture of the telescope. It is convenient to evaluate the coupling by propagating the receiver complex beam pattern backwards from the waveguide port to the telescope aperture, where the overlap integral with the incoming plane wave can be evaluated (Robieux 1959).

To realize the transition from waveguide to free-space propagation, we use corrugated conical horns (Thomas 1978) whose aperture has an amplitude distribution $E \propto J_{0}\left(\xi_{0} r / a\right)$, where $\xi_{0}$ is the first zero of the Bessel function $J_{0}$, and $a$ the aperture radius, the wavefront being spherical and centred at the apex of the cone. Such corrugated horns, widely used in millimetre waves, have the desirable properties of an aperture field that has azimuthal symmetry, is approximately constant over a full waveguide band, and has small cross-polarization. For the two lowest frequency bands (B1/B2), the phase deviation (from uniform) across the horn aperture is small enough to be neglected; for the highest frequency bands (B3/B4), and taking into account other design constraints, the phase deviation must be corrected by a small dielectric lens at the horn aperture.

The rest of the coupling optics must propagate (backwards, as mentioned above) the horn aperture field and map it to the telescope exit pupil with:

- a phase center at the Cassegrain focus;

- an amplitude distribution that realizes the best compromise between aperture efficiency, main beam efficiency, and sidelobe level, over the full frequency range of one band.

The design goal for the illumination at the edge of the pupil (socalled edge taper) was set at $14 \mathrm{~dB}$ below the bore sight level;

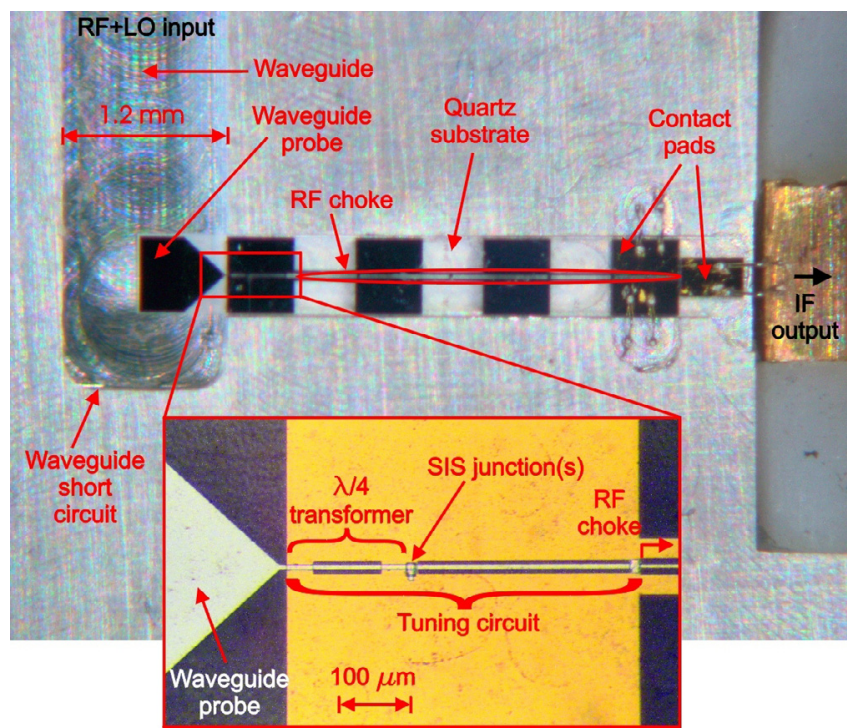

Fig. 8. Example of a mixer chip. See text for details.

this stems from a scientific requirement of a low sidelobe level, at the expense (compared with a $12 \mathrm{~dB}$ edge taper) of a slightly lower aperture efficiency.

Maintaining a constant illumination of the exit pupil over a finite bandwidth is possible with frequency independent optics, that must satisfy the simple condition that, considered in geometrical optics in the short wavelength limit, the horn aperture is imaged onto the telescope exit pupil (Chu 1983; Lamb 2003).

Our design for the relay optics uses two off-axis elliptical mirrors, that meet the above design conditions.

\subsection{Polarization diplexing}

For each frequency band, the separation of the $\mathrm{H}$ and $\mathrm{V}$ linear polarizations (with reference to their orientation in the Nasmyth cabin reference frame) is realized by a wire grid, part of the cold optics, and consisting of gold plated tungsten wires, $25 \mu \mathrm{m}$ in diameter with a pitch of $100 \mu \mathrm{m}$ (QMC Instruments 2007). To minimize the cross polarization induced by that separation, the wires are oriented perpendicular to the plane of incidence (Chu et al. 1975).

\section{Mixers and signal electronics}

All EMIR mixers are based on superconducting (SIS) tunnel junctions as mixing elements. IRAM employs $\mathrm{Nb}-\mathrm{Al} / \mathrm{AlOx}-\mathrm{Nb}$ junctions with typical sizes of about $1 \mu \mathrm{m}^{2}$ (Péron \& Schuster 2001; Krebs et al. 2007). These SIS junctions are deposited together with a superconducting circuit onto a quartz substrate. Figure 8 shows as an example the photograph of the Band 1 mixer chip. The superconducting circuit consists of different parts, each having a particular purpose: the waveguide probe on the left-hand side transforms the incoming radiation from waveguide mode to planar transmission line modes. The tuning circuit compensates for the junction capacitance and matches to the probe impedance and the RF choke blocks the RF radiation and only lets pass the IF signal. The contact pads finally, allow to connect the mixer chip by bonding to the ground and to the IF output, respectively.

The chip is placed in a channel perpendicular to the waveguide axis and stretches partly into the waveguide (see photograph). For double-sideband (DSB, see below) mixers, as shown 


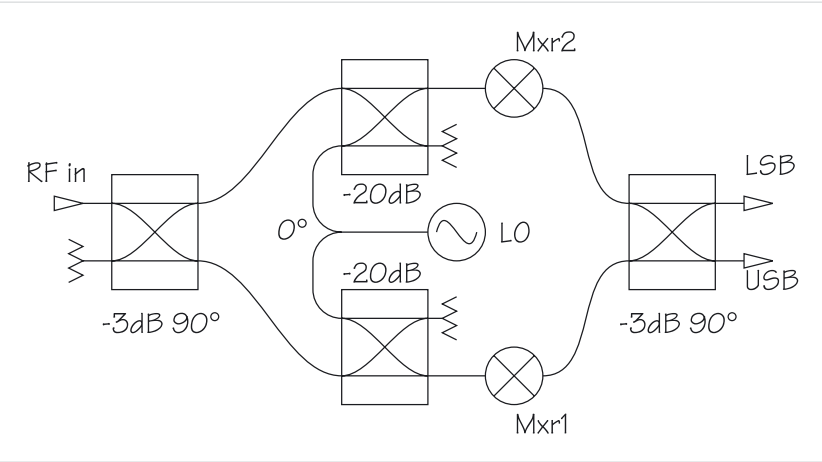

Fig. 9. Schematic view of a sideband-separating mixer.

in the picture, the length of the waveguide behind the chip is approximately a quarter wavelength, which allows matching to a large frequency range.

\section{Mixer types}

In heterodyne downconversion, the IF frequency is the difference between the LO and RF frequencies; two distinct RF frequencies result in the same IF frequency; one, the scientific target, is called the signal frequency while the other is called the image frequency. Either of these can be above or below the LO frequency, respectively called upper (USB) or lower (LSB) sideband.

This is the mode of operation of double sideband (DSB) mixers, which has several drawbacks, e.g. ambiguity in spectral line identification, and addition of atmospheric thermal noise from the atmosphere.

In single sideband (SSB) mixers, the response from the image sideband is (ideally) suppressed.

Sideband separating mixers (2SB) posess two distinct IF outputs, where the mixing products from the USB and LSB are separately present.

Each of these mixer types is present in some form in the system under discussion.

\subsection{Band 1}

Band 1 is equipped with the most recent development amongst the EMIR mixers: a sideband-separating mixer with very large IF band developed within the European project AMSTAR (Maier et al. 2007, 2008). A schematic view of a sidebandseparating mixer is shown in Fig. 9. Two double sideband mixers are connected at their inputs and outputs to quadrature hybrids. The LO signal is split and applied in-phase to the mixers through LO couplers. Since upper and lower sidebands undergo different phase shifts, they appear separately at the two outputs of the IF quadrature hybrid (Maas 1993).

For the EMIR Band 1 mixer the RF quadarature coupler, LO couplers and DSB mixers have been combined into one unit and realized as an E-plane split-block. Figure 10 shows a photograph of one half of such a block with mounted mixer chips. The two IF outputs are connected through two semi rigid cables to a commercially available IF $90^{\circ}$ hybrid coupler (Pasternack Enterprises 2009b, see Fig. 11). For details of the mixer design see Maier et al. (2007).

The RF frequency range of the 2SB mixer extends from 78 to $121 \mathrm{GHz}$ and the mixer covers an IF band from 4 to $12 \mathrm{GHz}$. Figure 12 above shows noise temperatures measured in the IF

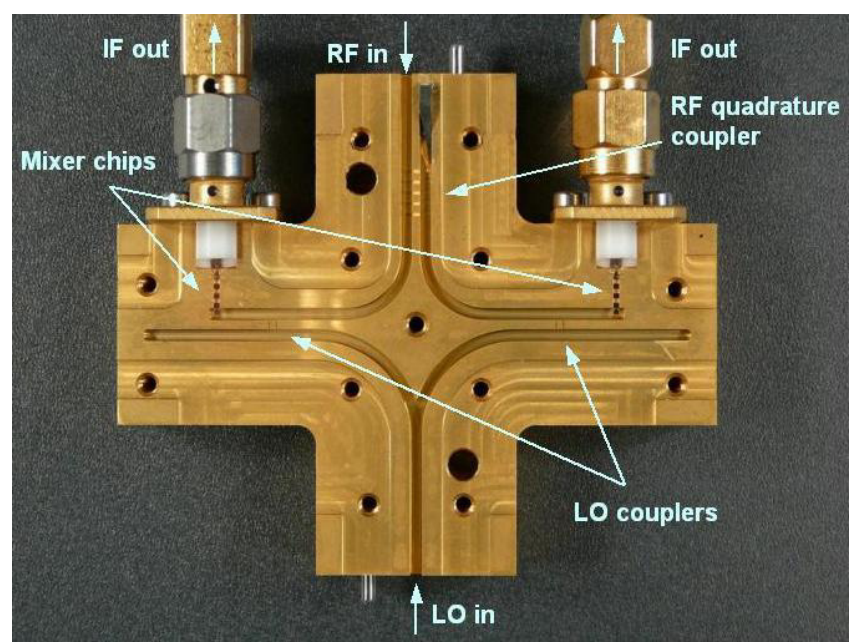

Fig. 10. Photograph of one half of the E-plane splitblock of Band 1 combining RF and LO couplers and SIS mixers.

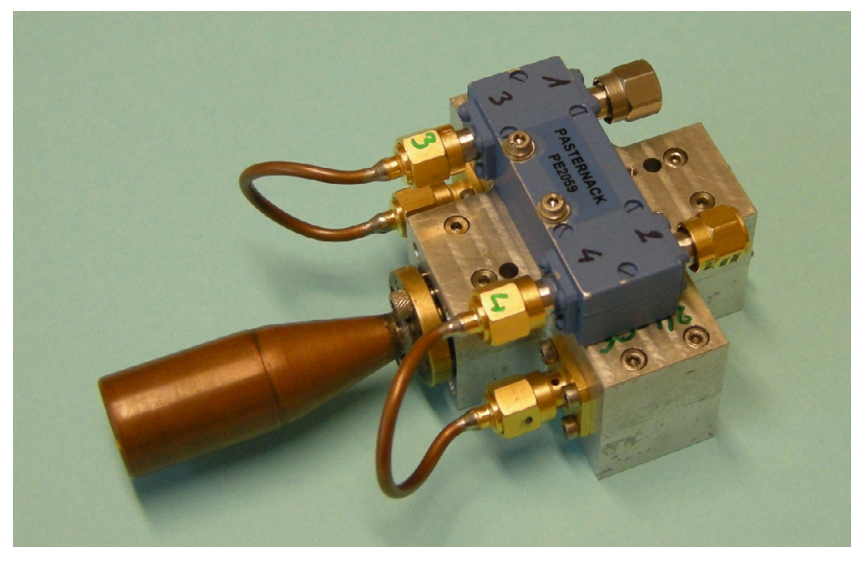

Fig. 11. Photograph of the Band 1 2SB mixer. The corrugated horn is seen on the left; the output IF quadrature coupler sits on top of the mixer block.

band for LO frequencies from 80 to $115 \mathrm{GHz}$. The obtained image rejection is shown in Fig. 12 below. The obtained values are almost always better than $-10 \mathrm{~dB}$ and the average lies around $-16 \mathrm{~dB}$.

\subsection{Band 2 and 3}

Bands 2 and 3 employ single-sideband mixers. In this type of mixer a movable backshort is located in the waveguide behind the mixer chip. The phase versus frequency dispersion of the round trip between the junction and the backshort is exploited to realize simultaneously a match at the signal frequency and the rejection of the image frequency.

The photograph in Fig. 13 shows the Band 2 mixer. Details of the Band 2 mixer design can be found in Navarrini \& Lazareff (2003). For details of the Band 3 mixer see Maier et al. (2005b).

Both mixers deliver one IF output of 4-8 GHz. Since the backshort position can only be optimized for the rejection of a distinct frequency, image rejection is a critical issue for backshort tuned SSB mixers, especially for these large IF bands. Figure 14 shows as an example the image rejection obtained with the Band 3 mixer for an LO frequency of $231 \mathrm{GHz}$ and USB operation. Since the backshort position is set to optimize the rejection of the center frequency, the best values are obtained in the 

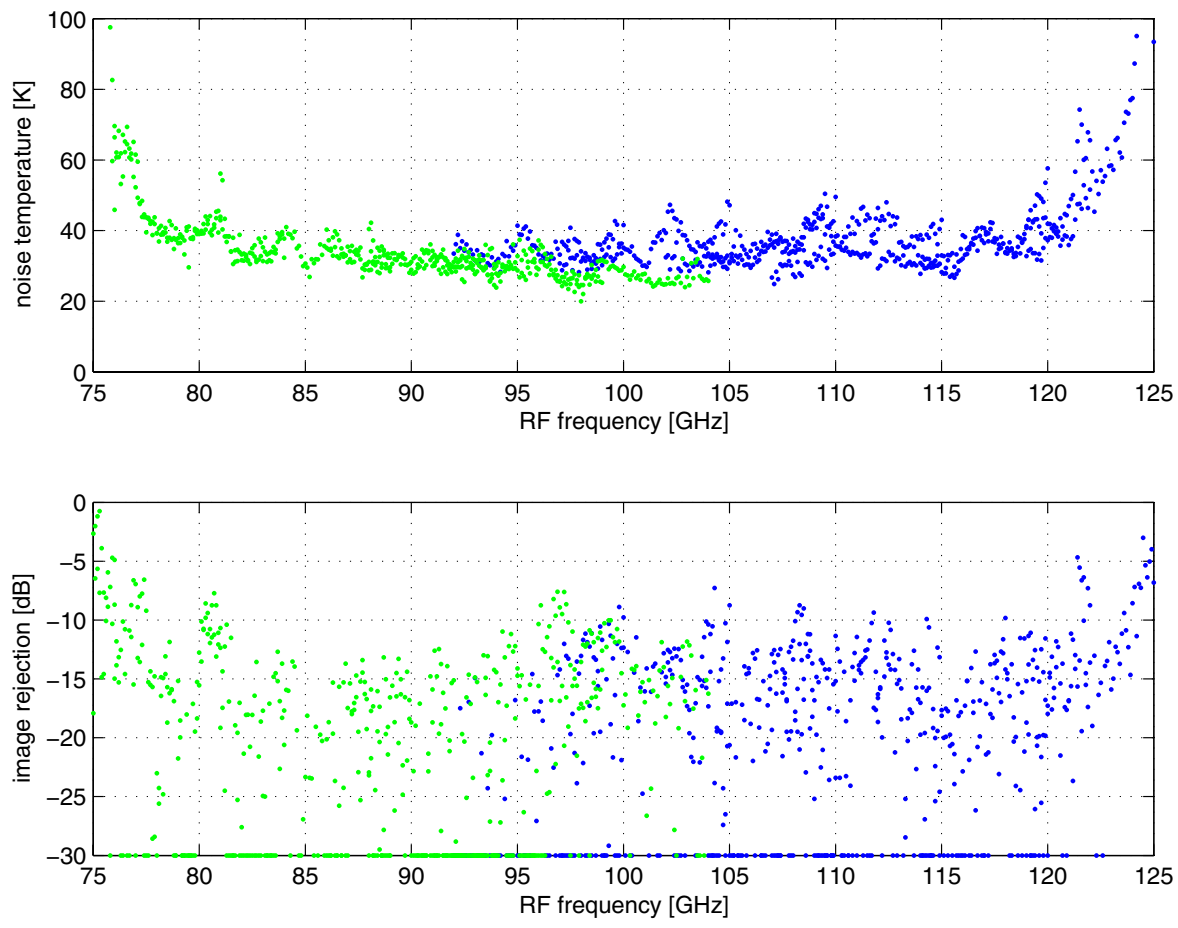

Fig. 12. Band 1 noise temperatures (top) and image rejection (bottom) measured in the IF band for different LO frequencies. LSB measurements are plotted in green, USB results are shown in blue. Measurements performed in the laboratory, with an automated test set, before the mixer was integrated into the receiver. Note that the range of RF frequencies measured and displayed goes beyond the design range.

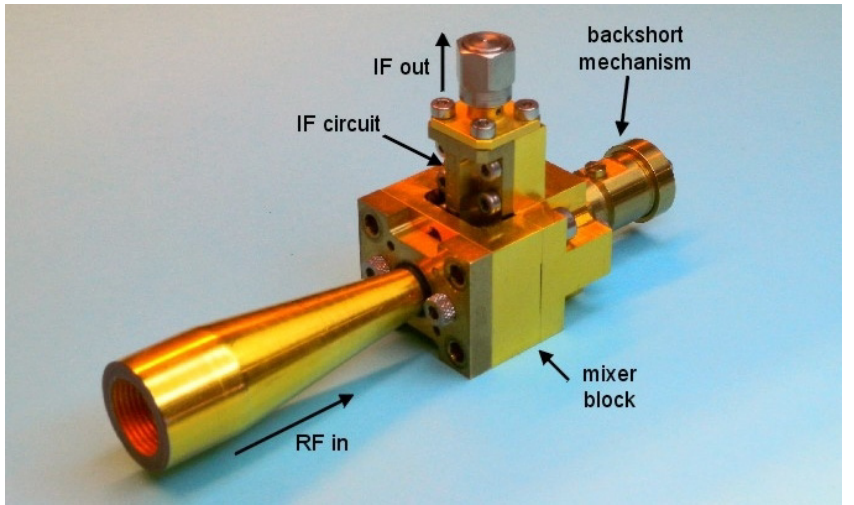

Fig. 13. Photograph of the Band 2 mixer.

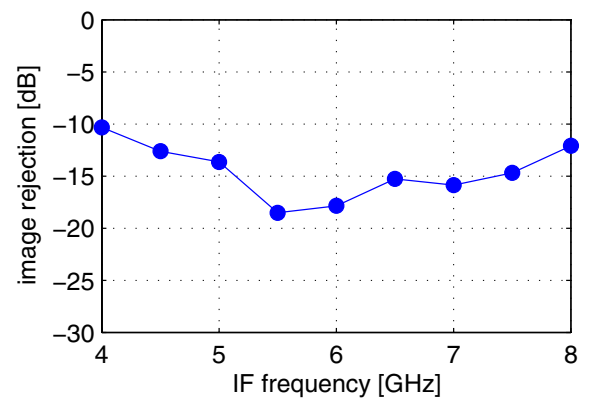

Fig. 14. Image rejection of the Band 3 mixer measured for USB operation at $f_{\mathrm{LO}}=231 \mathrm{GHz}$.

middle of the band. Nevertheless, rejections better than $-10 \mathrm{~dB}$ are reached even at the band edges.

\subsection{Band 4}

The sideband-separating mixer employed in Band 4 has been originally developed for the ALMA Band 7 cartridge

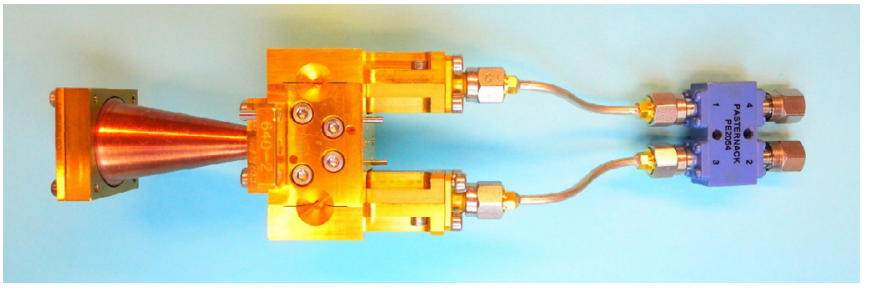

Fig. 15. Photograph of the Band 4 mixer.

(Maier et al. 2005a) and tuned down in frequency to match the EMIR Band 3 frequency range. Since the ALMA mixer had to be produced in a small series production, emphasis had been laid on an easy and reliable fabrication. Therefore a modular approach has been chosen allowing the test of the individual components of the $2 \mathrm{SB}$ mixer prior to integration and thus reducing possible causes of failures. A photo of the EMIR Band 4 mixer is shown in Fig. 15. It consists of the coupler block combing the $90^{\circ} \mathrm{RF}$ hybrid coupler and both LO couplers (cf. above), two DSB mixers and the IF hybrid coupler (Pasternack Enterprises 2009a).

The ALMA mixer has been adapted to the lower frequency range of Band 4 by changing the RF coupler and increasing the size, and thus the capacitance, of the SIS junctions.

Noise temperatures measured in the IF band for LO frequencies between 266 and $359 \mathrm{GHz}$ are shown in Fig. 16 (top). The image rejection is always better than $-10 \mathrm{~dB}$ and the average lies around $-17 \mathrm{~dB}$ as can be seen in the plot in Fig. 16 (bottom).

\subsection{IF amplification}

The outputs of the mixers, in the intermediate frequency (IF) band must be amplified to a level suitable for further processing, and safely above the room temperature thermal noise $(k T \mathrm{~W} / \mathrm{Hz}$ ). The first active element in the IF chain is a cryogenic low noise amplifier (LNA) designed by Yebes Observatory, Spain (Lopez-Fernandez et al. 2006). This amplifier provides $\approx 35 \mathrm{~dB}$ of gain with an input-referred noise temperature of $\approx 4 \mathrm{~K}$. 

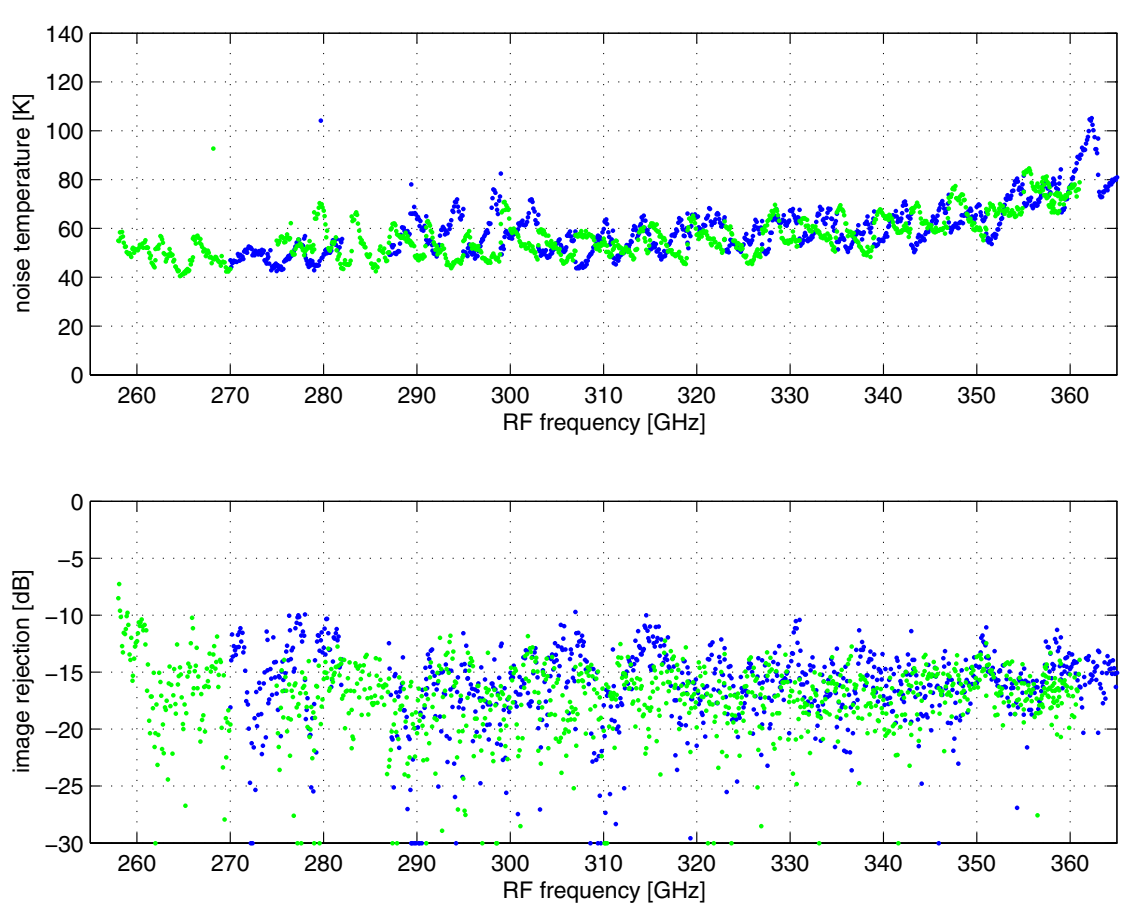

Fig. 16. Band 4 noise temperatures (above) and image rejection (below) measured in the IF band for different LO frequencies. LSB measurements are plotted in green, USB results are shown in blue.
Between an IF output port of a mixer and the input of the LNA, an isolator is inserted to suppress standing waves that would cause gain ripple across the bandpass. Further amplification is provided by room temperature amplifiers.

\section{Local oscillator}

For each of the four bands, the local oscillator (LO) power is generated in a commercial Gunn oscillator (Carlstrom J.E. Co. 2007), mechanically tuned (under remote control) and phase referenced to a commercial microwave oscillator covering a $f_{1} \div f_{2} \simeq 1.3$ band located between 60 and $120 \mathrm{GHz}$. For band 2,3 , and 4 , the signal from the Gunn is fed to a varactor multiplier (Virginia Diodes Inc. 2007) with multiplication factor of $\times 2, \times 3$ and $\times 3$ respectively. The LO signal at the operating frequency is carried across the back of the cryostat through a vacuum feedthrough, in a thin-walled stainless steel waveguide, whose inside is gold plated to minimize ohmic losses. To further minimize LO transmission losses, the waveguide for bands 2, 3 and 4 is oversize; bends are avoided or carefully dimensioned to avoid mode conversion and associated resonances and power dips. For Band 4, the varactor tripler is located inside the cryostat, in close proximity to the mixers; this results in higher efficiency and optimum drive level.

In the vicinity of each mixer pair, the LO signal is split evenly. For bands 2 and 3, the LO enters an injection coupler. The coupling is kept as small as the available LO power allows $(\simeq-17 \mathrm{~dB})$ to minimize the noise added to the signal path. For bands 1 and 4, that use image separating mixers, the LO is fed directly to the mixer, inside which the LO coupling is integrated. Even though the relative $\mathrm{LO}$ power of the two ( $\mathrm{V}$ and $\mathrm{H}$ polarization) SIS mixers is fixed, careful component screening and the tolerance of such mixers to the LO drive level allow a satisfactory operation.

\section{Cryogenics}

The cryostat is of cylindrical shape, $60 \mathrm{~cm}$ in diameter. A three stage Gifford-McMahon cryocooler Sumitomo SRDK-3ST Type 6 (SHI-APD Cryogenics Europe 2007) provides cooling power to the radiation shields $(70 \mathrm{~K}, 15 \mathrm{~K})$, the cold optics and the first stages of IF amplification $(15 \mathrm{~K})$, the mixers and their output IF isolators (4 K). Fiberglass reinforced epoxy (G11) structures provide mechanical support for the cold payload, with suitable rigidity, compliance to differential thermal contraction, and minimal heat flow. Cooldown curves are shown in Fig. 17.

\section{Monitor and control}

A dedicated workstation, receiving high-level commands, runs algorithms for setting the local oscillator frequencies, tuning the SIS mixers, and moving optical elements involved in frequency diplexing or calibration. To optimally use the heritage of past developments, two technologies are jointly used for interfacing the workstation to the receiver hardware. CAN-bus modules are used to interface the local oscillators and, generally, motors: mixer backshorts (when applicable) and optical elements. I2C modules provide precision interfaces to analog voltages; the I2C bus(es), accessed through a CAN-I2C bridge, can be put in "sleep" mode during observations to eliminate digital cross-talk to critical analog bias voltages. Almost all CAN or I2C modules were custom-designed to meet the system's needs. The various functions are listed in Table 3.

\section{System performance}

Following assembly and verification in the Grenoble laboratories, the EMIR system was installed in the receiver cabin of the 30-m telescope (Pico Veleta, Sierra Nevada, Spain) in March 2009. See Fig. 18. 


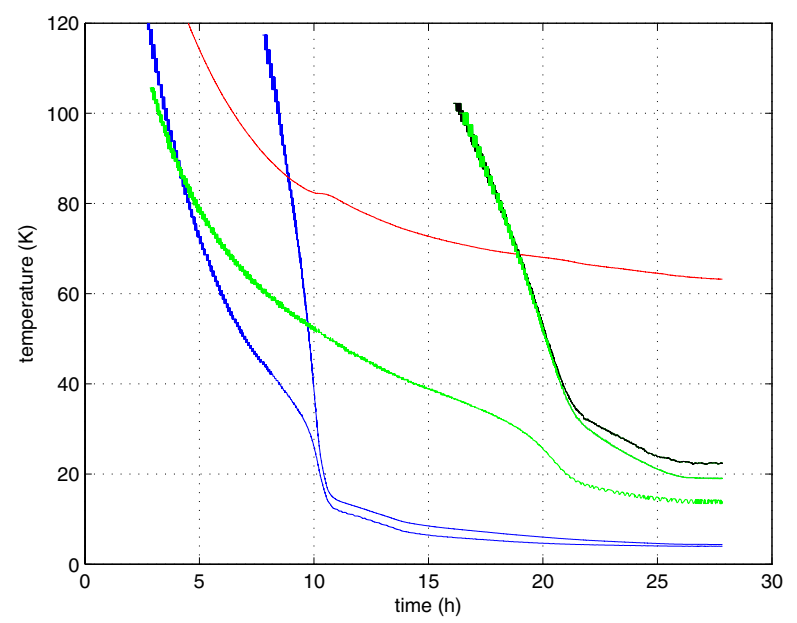

Fig. 17. Cooldown of the cryostat and its payload. Blue, left to right: cryocooler $4 \mathrm{~K}$ head; one of the Band 1 mixers. Green, left to right: cryocooler $15 \mathrm{~K}$ head; one of the low noise IF preamplifiers. Black: one of the internal cold calibration loads. Red: cryocooler $70 \mathrm{~K}$ cold head.

Table 3. Monitor and control functions implemented in the EMIR system.

\begin{tabular}{lcccc}
\hline \hline Subsys & Function & Ch/mod & Mods & Type \\
\hline Opt. & Carousels & 1 & 2 & Step mot \\
& Dichroics & 1 & 1 & Step mot \\
& Ambient Temp & 1 & 2 & Temp sens \\
\hline Mxrs & Junction bias & 4 & 4 & D/A + A/D \\
& Josephson coils & 4 & 2 & D/A + A/D \\
\hline LO & Gunn osc tuning & 2 & 4 & DC servo \\
& Attenuators & 3 & 4 & DC servo \\
& Harm mxr bias & 1 & 4 & D/A + A/D \\
& PLL IF level & 1 & 4 & A/D \\
& Gunn bias & 1 & 1 & D/A + A/D \\
& PLL err volt & 1 & 4 & A/D \\
& PLL loop gain & 1 & 4 & D/A + A/D \\
\hline IF & Bias monitor & 4 & 4 & A/D \\
& Attenuator & 4 & 4 & 4-bit I/O \\
& IF level & 4 & 4 & A/D \\
\hline Cryo & Temp sensors & 8 & 1 & A/D \\
& Vacuum gauge & 1 & 1 & A/D \\
\hline
\end{tabular}

Notes. Some abbreviations have been used because of width limitations. Step mot: stepper motor; Temp sens: temperature sensor; Mxrs: SIS mixers; D/A: digital to analogue converter (and conversely); Gunn osc: Gunn oscillator; DC servo: DC servomotor; Harm mxr: harmonic mixer; PLL: phase lock loop; err volt: error voltage. "Josephson coils" is shorthand for the coils that generate a magnetic field to suppress the Josephson effect in the superconducting junctions.

\subsection{Receiver noise}

Following the integration of the receiver, systematic measurements of noise were performed in the laboratory. The results shown in Fig. 19 were obtained in single band mode, without the dichroic filters. The noise measurements are made on the Rayleigh-Jeans scale, which amounts (Kerr 1999) to excluding the vacuum fluctuations from the definition of receiver noise. Using the dichroic filters for dual frequency observations carries a noise penalty which is band- and frequency-dependent, amounting to typically $10-15 \mathrm{~K}$. The noise performance for Band 1 is in good agreement with the mixer measurements shown in Fig. 12. For Band 4, an excess of receiver noise is

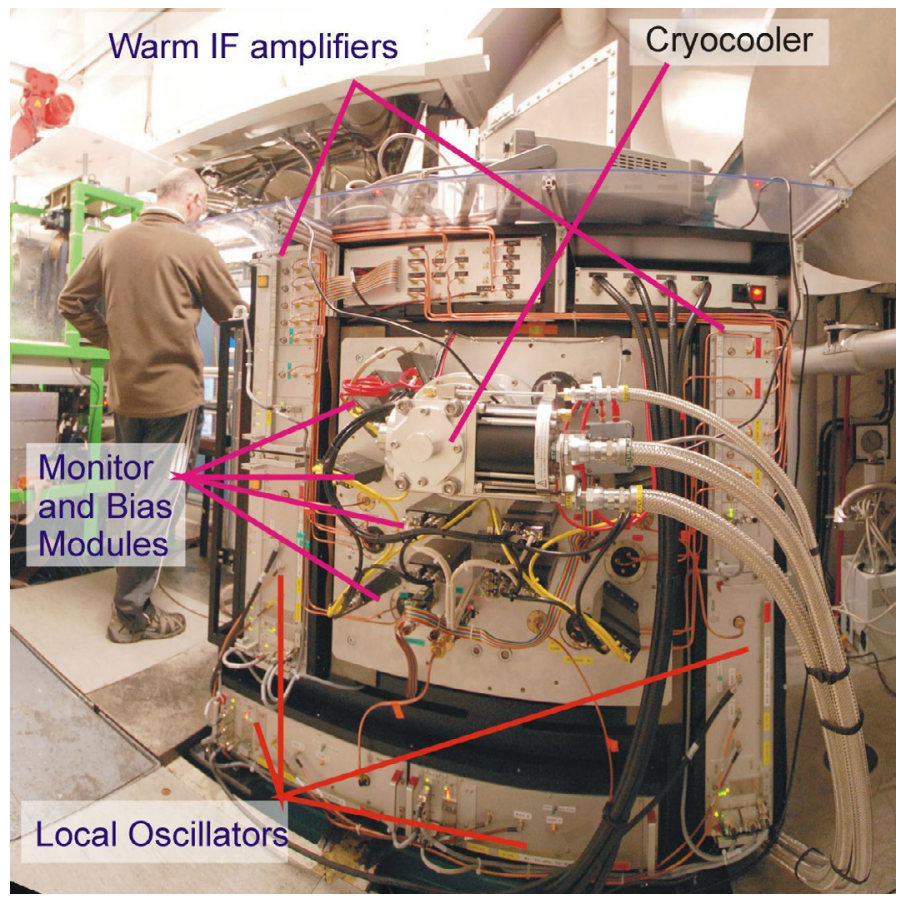

Fig. 18. The EMIR system in the 30-m telescope receiver cabin. Besides the annotated elements, one can see, on the right, part of the Nasmyth mirror that maps the telescope axis to a fixed reference frame, and on the left, the HERA $1.3 \mathrm{~mm}$ band $2 \times 9$, beam receiver as well as one of the project team members.

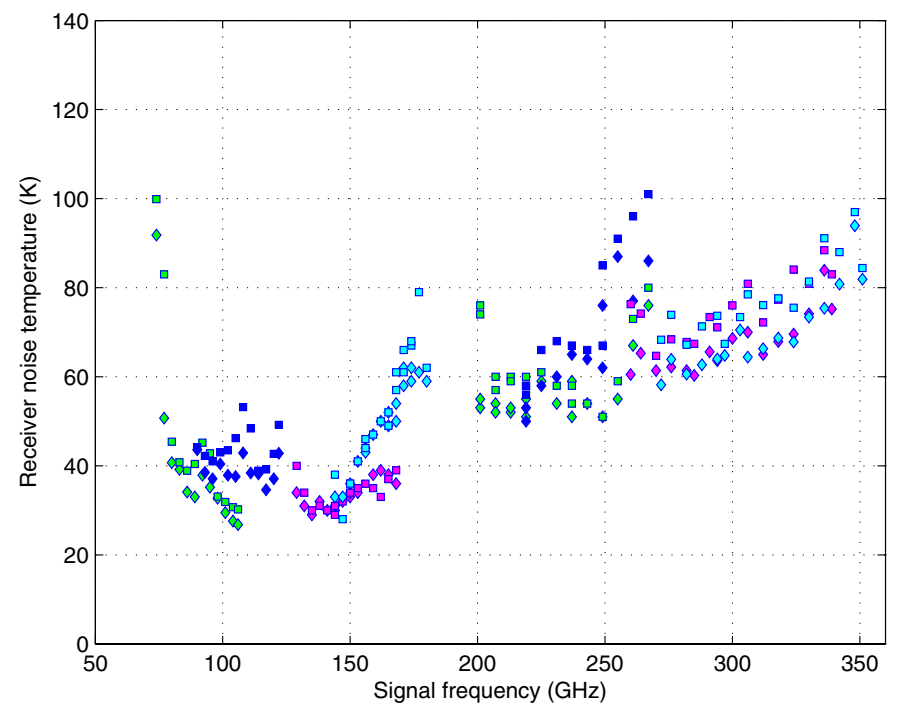

Fig. 19. Receiver noise temperature versus signal frequency, measured in the laboratory using the hot-cold method. Squares: H polarization; diamonds: V polarization. Bands 1 and 3: green: LSB, blue: USB; bands 2 and 4: magenta: LSB, cyan: USB.

present at the upper end of the band, compared with the mixer measurement (Fig. 16). The image band rejection was measured after mixer fabrication, but not after system integration; we assume that this mixer property is not significantly affected by signal optics. It is in principle possible, for observations where a very accurate line calibration is necessary, to measure the image band rejection by a method that is free from any assumption. 


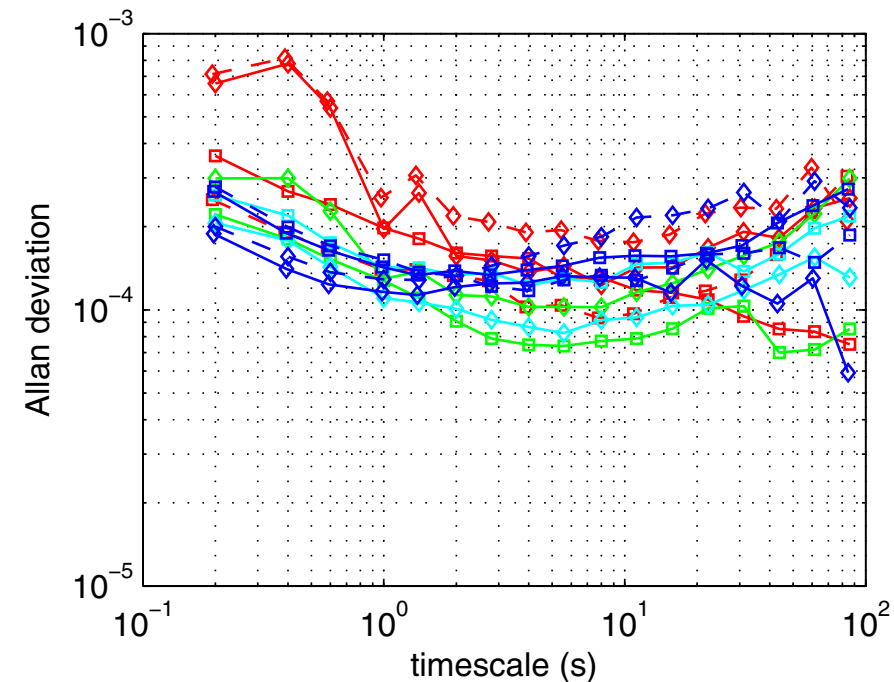

Fig. 20. Allan deviation versus time scale for the receiver IF output power (normalized to mean value). Frequency band is color coded. Band 1 (101 GHz LO): red; Band 2 (150 GHz LO): green; Band 3 (237 GHz LO): cyan; Band 4 (300 GHz LO): blue. Polarization: H: squares; V: diamonds. Sideband channel (when applicable) LSB: continuous; USB: dashed.

\subsection{Total power stability}

Total power stability is not a priori an essential specification for a spectral receiver, because it is expected that nearly identical fluctuations will affect both the line frequency range and the adjacent (so-called baseline) regions, that are used to establish the zero point of the spectral power scale. However, a poor stability may be an indicator of second-order effects that invalidate the above mentioned assumption. Total power stability is also important when performing pointing measurements on continuum sources (planets, quasars, etc.).

Total power stability was measured with the receiver staring at a room temperature matched load. An Agilent E4419B dual-channel power meter, with two E9300A power sensors, was connected to the warm IF outputs of the $\mathrm{H}$ and $\mathrm{V}$ polarization channels of one frequency band and sideband combination. Measurements were acquired with a $200 \mathrm{~ms}$ sampling time for a duration of $600 \mathrm{~s}$. The time sequences were processed to produce the Allan deviation $(\sqrt{\text { variance }})$ versus timescale. The results are displayed in Fig. 20. Apart from the anomalous behaviour of Band $1, \mathrm{~V}$ polarization at short timescales, the Allan deviation is always less than $4 \times 10^{-4}$; a typical value overall is $2 \times 10^{-4}$.

The same data were processed by Fourier transform, better suited to reveal sinusoidal perturbations in the total power stability, as might be caused (whether mechanically or thermally) by the reciprocating cryocoler. A line is indeed present in the frequency domain at $1 \mathrm{~Hz}$, with an amplitude (normalized to the mean value) typically $0.5-3 \times 10^{-4}$, with a maximum value $9 \times 10^{-4}$ (Band 1, USB, V polarization).

\subsection{On-sky alignment}

The alignment of the receiver as a whole is taken care of by suitable pointing offsets (as long as it remains within a fairly large region of the focal plane where aberrations are negligible); therefore we discuss only internal co-alignment of the Emir system.
Table 4. Lateral misalignments between frequency band pairs (used in dual frequency mode).

\begin{tabular}{lccc}
\hline \hline Band pair & B1/B2 & B1/B3 & B2/B4 \\
\hline Misalignment & $2^{\prime \prime}$ & $1^{\prime \prime}$ & $0.3 \pm 0.2^{\prime \prime}$ \\
\hline
\end{tabular}

Table 5. Lateral misalignments between polarizations.

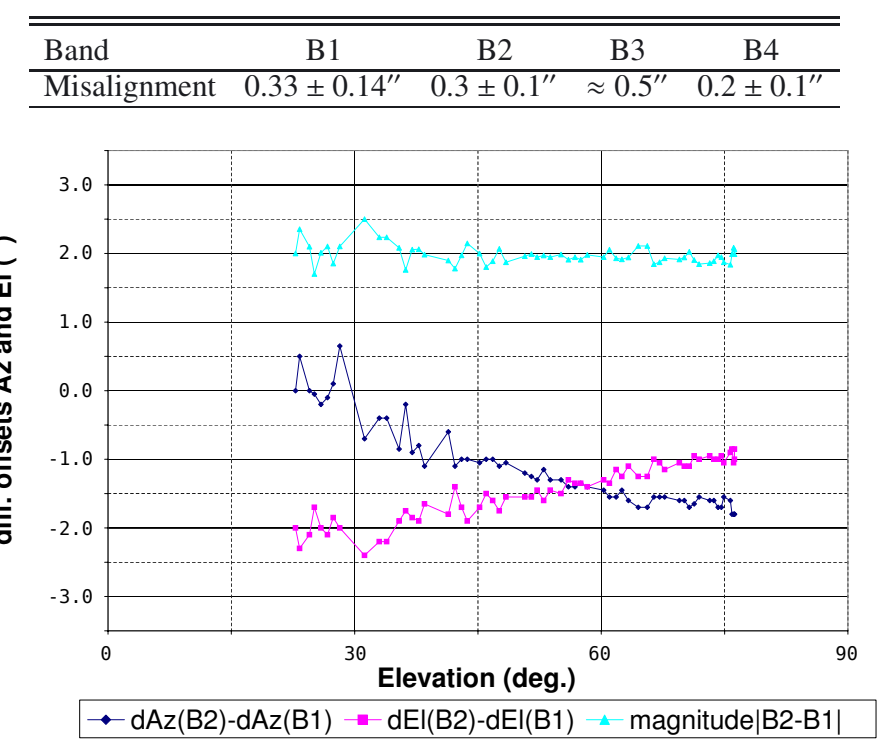

Fig. 21. Measurements of misalignment between Band 1 and Band 2 . Because of the Nasmyth folding mirror, a misalignment originating in the receiver rotates on the sky as a function of the elevation. While the azimuth (blue) and elevation (magenta) components of the on-sky misalignment are elevation-dependent, the magnitude (cyan) remains constant. Note the increased scatter at low elevation due to atmospheric seeing.

\section{Lateral alignment}

A good lateral alignment of the receiver feeds in the focal plane ensures that the beam pattern of two receiver channels are coaligned on the sky. One should further distinguish:

- lateral co-alignment between different frequency bands. This is important for dual-frequency observations, especially in observations of point sources, and in mapping mode (in the latter case, offsets can be corrected if the map is fully sampled. The worst case misalignment is $2^{\prime \prime}$;

- lateral co-alignment of polarization channels. Besides what was mentioned above, co-alignment of polarization channels is critical in polarimetric observations to avoid spurious couplings between Stokes parameters and spatial dopplerinduced gradients. The worst-case misalignment is $0.5^{\prime \prime}$.

In both cases, one would like any mis-alignment to be a small fraction of the beam width, which is, expressed in arc seconds: $\approx 2.06 \times 10^{5} \lambda / D$, where $\lambda$ is the observing wavelength, and $D=$ $30 \mathrm{~m}$ the telescope diameter; e.g. 10.5" at $230 \mathrm{GHz}$. Results are shown in Tables 4 and 5.

\section{Longitudinal (focus) alignment}

As in the case of lateral alignment, the absolute alignment is not critical, apart from the limits set by the onset of spherical aberrations, which are not significant in the present discussion. 


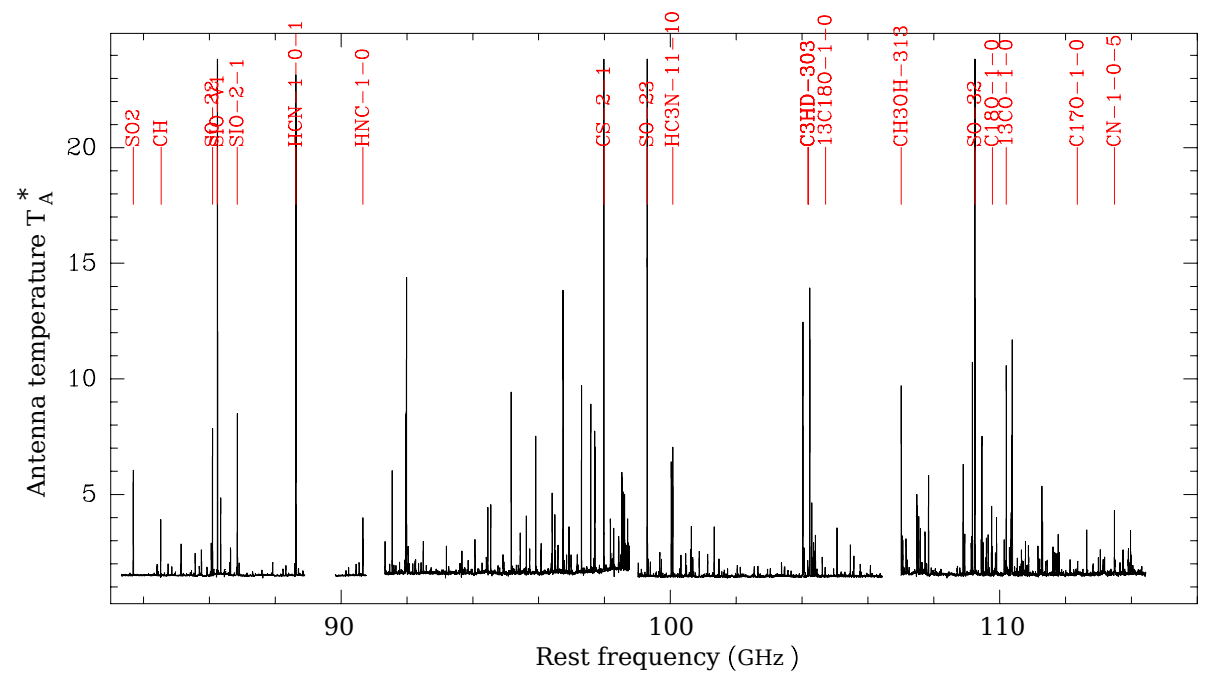

Fig. 22. Line spectrum of the Orion molecular core taken during commissioning of EMIR in April 2009. Almost $32 \mathrm{GHz}$ of RF frequency space can be surveyed with just two local oscillator settings.
Table 6. Longitudinal misalignments between bands.

\begin{tabular}{cccc}
\hline \hline Band pair & B1/B2 & B1/B3 & B2/B4 \\
\hline & $-0.30 \mathrm{~mm}$ & $-0.25 \mathrm{~mm}$ & $-0.35 \mathrm{~mm}$ \\
\hline
\end{tabular}

When two channels (whether frequency or polarization) are observed simultaneously, each one will have an optimum focus; the measurements are affected by a common time-dependent focus shift originating in the thermal behavior of the main reflector: only the differential measurement is significant for the receiver. Optimum focus is achieved by an axial displacement of the Cassegrain (secondary) mirror; the corresponding displacement of the Cassegrain focus is larger by a factor $M^{2} \simeq 773$, where $M=27.8$ is the Cassegrain lateral magnification (Baars et al. 1993). And again, we distinguish between:

- longitudinal co-alignment between different frequency bands. Results are shown in Table 6 . The worst case focus discrepancy is between B2 and B4, and is $\simeq 0.35 \mathrm{~mm}$ in secondary mirror travel;

- longitudinal co-alignment of polarization channels. Any deviation is negligible, i.e. $<0.05 \mathrm{~mm}$ in secondary mirror travel.

To quantify the impact of the measured longitudinal misalignment(s), we examine the worst case: B2/B4. We scale (by $M^{2}$ ) the $0.35 \mathrm{~mm}$ secondary mirror displacement (B2-B4) to $270 \mathrm{~mm}$ displacement of the Cassegrain focus. The coupling loss from longitudinal misalignment is given by:

$K_{\text {long }}=\frac{1}{1+\frac{1}{4}\left(\Delta z / z_{0}\right)^{2}}$

(Goldsmith 1992), where $z_{0}$, the confocal distance, is $389 \mathrm{~mm}$ at $\lambda=2 \mathrm{~mm}$ and $175 \mathrm{~mm}$ at $\lambda=0.9 \mathrm{~mm}$. Distributing the focus error in proportion to wavelength results in a common value of of the coupling efficiency: $95 \%$. Note that this loss applies only to point sources, not to observations of extended sources, which will only be affected by a minor beam broadening. Performing the same analysis for the B1/B3 pair results in $98.7 \%$ efficiency.

\subsection{Forward efficiency}

Forward efficiency is the fraction of the receiver's power radiation diagram coupled to the sky, as opposed to coupling to the
Table 7. Measured forward efficiencies in the four bands.

\begin{tabular}{lcc}
\hline \hline Band & Freq. $(\mathrm{GHz})$ & $F_{\text {eff }}$ \\
\hline 1 & 86,89 & 0.95 \\
$2(1)$ & 147 & 0.92 \\
$2(3)$ & 145,147 & 0.95 \\
3 & 210,230 & 0.94 \\
3 & 260 & 0.88 \\
4 & 330 & 0.86 \\
\hline
\end{tabular}

Notes. Numbers in parenthesis refer to the alternate band configured in dual-band mode.

ground and ohmic losses in telescope optics. It is a joint property of the receiver and the telescope. The forward efficiencies reported in Table 7 were measured by the skydip method and are referred to an interface between receiver and the telescope defined by the hot/cold load calibration plane.

\section{Astronomical results}

Figure 22 displays data taken shortly after installation and demonstrates the wide band capability of the Band 1 mixer; the displayed spectrum was obtained in just two local oscillator settings, thanks to the dual (USB+LSB) IF sidebands, each covering 4-12 GHz. Figure 23 shows a line survey of the molecular sources IKTau and QH231, and demonstrating the scientific benefits of wide instantaneous frequency coverage. Figures 24 and 25 further illustrate this point: the high obscuration of SMM14009 did not allow obtaining an optical redshift; the redshifted lines $\mathrm{CO}(3-2)$ and $\mathrm{CO}(5-4)$ were detected following a blind search covering a wide frequency range. This is the first time, at least at the 30-m telescope, that a quasar redshift was determined ab initio from mm-wave observations. Finally, Fig. 26 illustrates that, despite being single-pixel, the Emir receiver's efficiency allows to map large areas, as in this $11^{\prime} \times 7.5^{\prime}$ map of M51 in the ${ }^{12} \mathrm{CO}(1-0)$ transition with a $21^{\prime \prime}$ beam.

\section{Conclusion}

We have designed, built, tested, and put in operation at the IRAM 30-m radiotelescope a multi-band, dual polarization heterodyne receiver. It covers most of the atmospheric transmission windows between $75 \mathrm{GHz}$ and $350 \mathrm{GHz}$. It offers a noise 

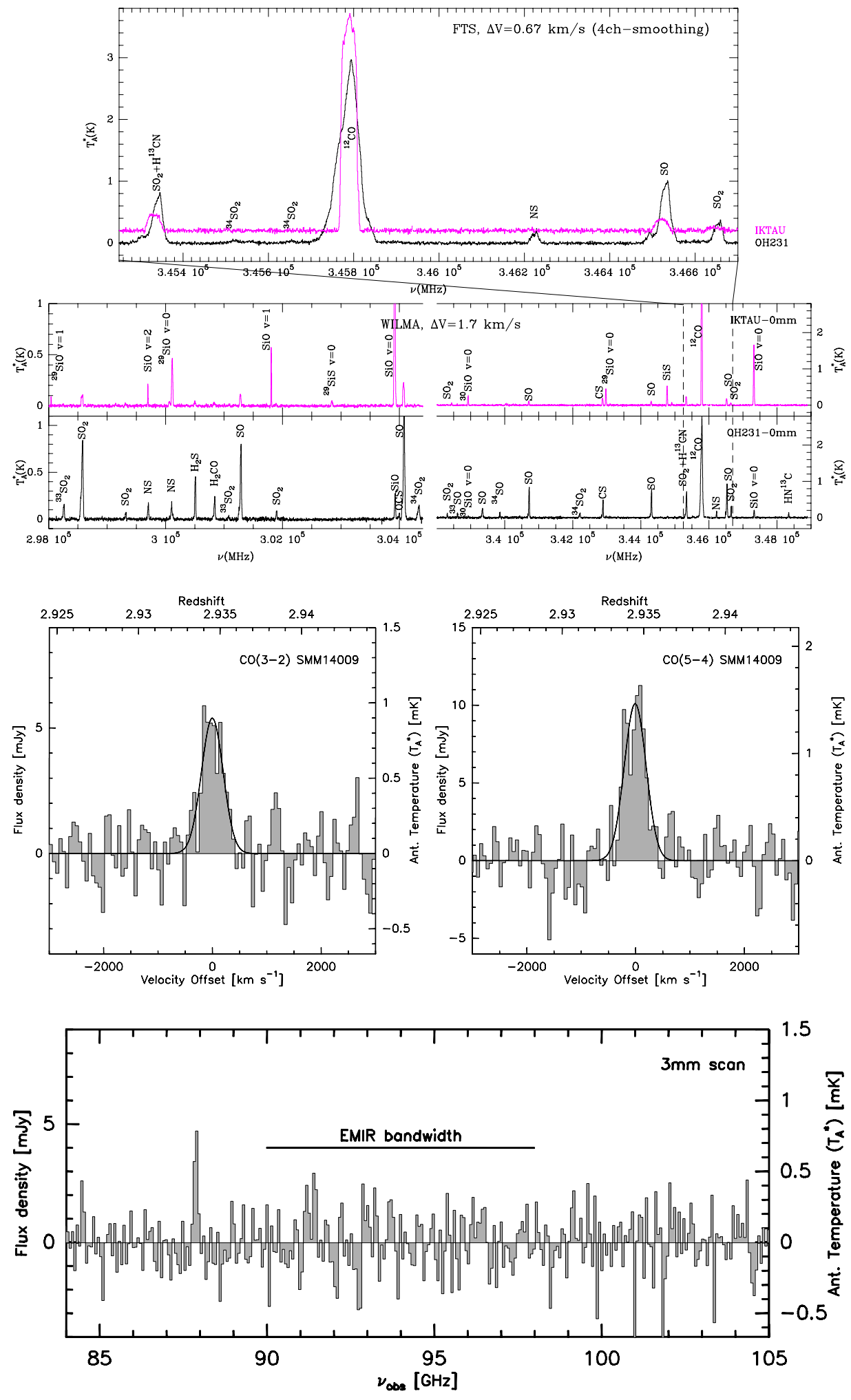

Fig. 23. Data subset of EMIR spectra at $0.9 \mathrm{~mm}$ of the O-rich stars IK Tau and OH 231.8+4.2 obtained in January 2011 as part of an on-going mm-wavelength spectral line survey of O-rich evolved stars by Sanchez Contreras et al. (2011). Bottom: selected spectra obtained with WILMA around 300 and $343 \mathrm{GHz}$. WILMA is a broad band spectrometer of the 30-m telescope of the autocorrelator type. Top: zoom of a smaller frequency range (indicated by vertical dashed lines in the bottom panels) showing higher spectral resolution spectra obtained with fast fourier transform spectrometers (FTS).

Fig. 24. SMMJ14009+0252, a distant starforming submm galaxy, is so heavily obscured by dust that no optical redshift could be measured. A $20 \mathrm{GHz}$ scan of the $3 \mathrm{~mm}$ atmospheric window allowed the detection of the redshifted $\mathrm{CO}(3-2)$ line, confirmed by the detection of $\mathrm{CO}(5-4)$ in the $2 \mathrm{~mm}$ window. From work by Weiß et al. (2009).

Fig. 25. Scan of a large part of the $3 \mathrm{~mm}$ atmospheric window, performed as part of the blind search for CO lines in SMMJ14009. This shows the noise level and baseline quality achieved. The redshifted $\mathrm{CO}(3-2)$ line appears at $\approx 87.7 \mathrm{GHz}$. Note: this spectrum is displayed at a coarser resolution compared with Fig. 24.

performance of a few times the "quantum limit" $h v / k$. The observing modes are flexible, including simultaneous dual polarization, a choice of single-frequency or dual-frequency (B1/B2, $\mathrm{B} 1 / \mathrm{B} 3, \mathrm{~B} 2 / \mathrm{B} 4$ ), and dual sideband (for B1, B4, and in the near future B3). The instantaneous aggregated bandwidth (including polarization diplexing) available for spectral analysis can be (depending on the selected configuration) up to $40 \mathrm{GHz}$ (64 GHz in the near future).
This receiver has a compact foot-print, is fully remote controlled, and has been in operation for more than two years with no maintenance.

EMIR has brought to the 30-m telescope and its community of users not only the possibility to do similar projects with better signal-to-noise, but has also enabled projects not possible before, due mainly to its broad frequency coverage, state-of-art noise performance, and multiplex capabilities. 


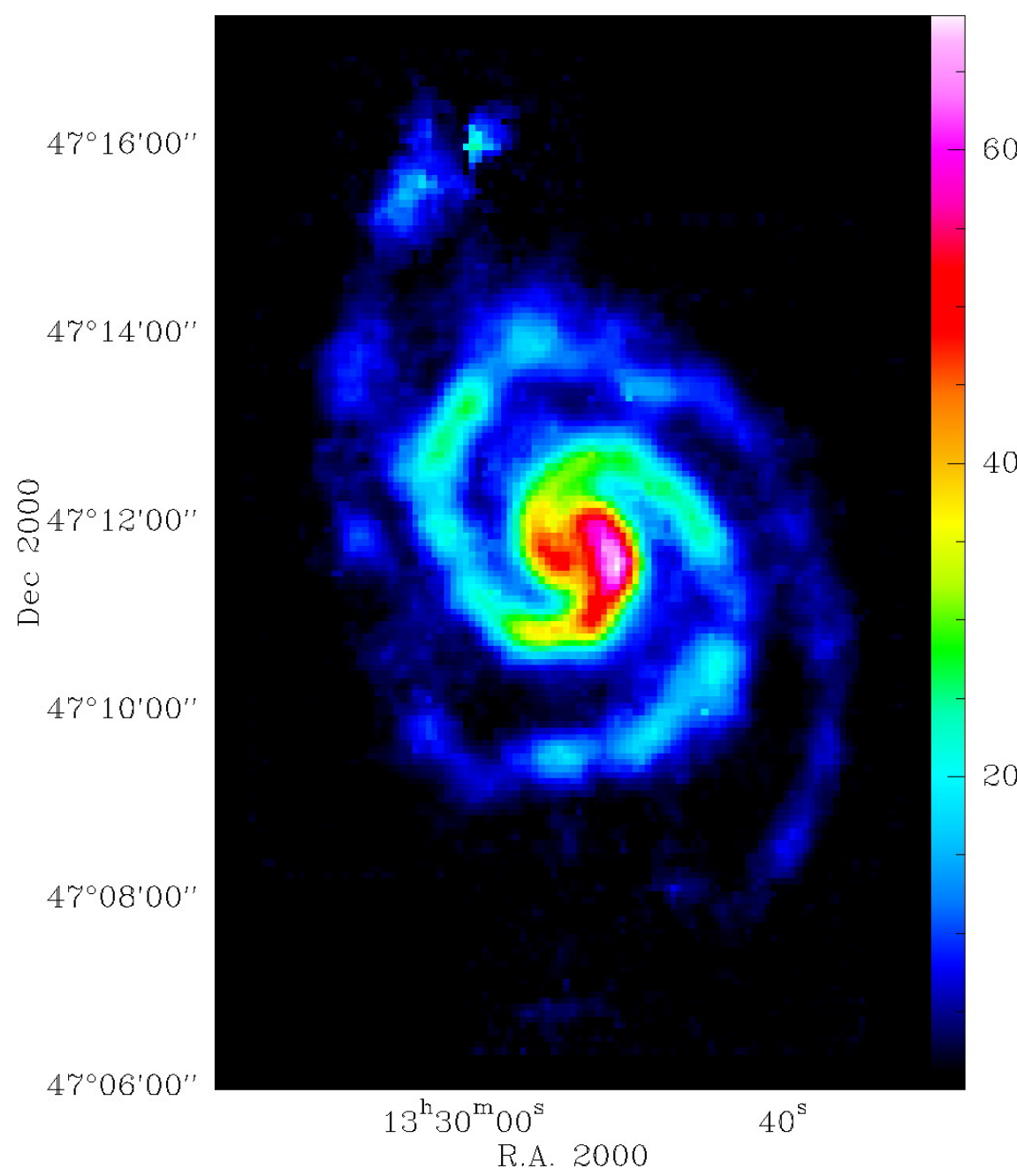

Fig. 26. Map of the galaxy M51, in the ${ }^{12} \mathrm{CO}(1-0)$ transition $(115.27 \mathrm{GHz}$ rest frequency). Courtesy of Pety and Schinnerer; data acquired as an IRAM large program for the PAWS project. The colors code the integrated (over radial velocities) line intensity $\int T_{\mathrm{mb}} \mathrm{d} v$, units $\mathrm{K} \mathrm{km} \mathrm{s}^{-1}$.
Acknowledgements. A number of IRAM staff members contributed their skillful and dedicated work to this project. A. Barbier, D. Billon-Pierron, and S. Halleguen fabricated and characterized the SIS junctions; Y. Boutglay, E. Naffetat, M. Parioleau, and J. Reverdy assembled and characterized the SIS mixers; F. Cope prepared CAD drawings for many mechanical parts and subsystems; L. Coutanson, J.-M. Daneel, and J.-L. Orecchia fabricated mixer blocks and other parts to exquisite tolerances; M. Berton, F. Coq, B. Pissard, and P. Serres assembled and characterized the system; W. Brunswig, G. Paubert, A. Sievers, H. Ungerechts, and H. Wiesemeyer played a significant role in integrating the new system into the 30-m telescope. We are grateful to J. D. Gallego, A. Barcia and their colleagues at Centro Astronomico Yebes, who designed the cryogenic IF amplifiers and supervised their industrial production. We are also grateful to C. Contreras, A. Weiss, and J. Pety for allowing us to use their scientific results as illustrations of the performance of EMIR. This work has benefited from research funding from the European Community's sixth Framework Programme under RadioNet R113CT 20035058187.

\section{References}

Baars, J., Greve, A., Hein, H., D., M., Penalver, J., \& Thum, C. 1993, Design parameters and measured performance of the IRAM 30-m millimeter radio telescope, Tech. Rep., 298

Carlstrom J. E. Co. 2007, Wide band Gunn oscillators, 5736, S. Harper Ave, Chicago, IL

Chu, T. 1983, IEEE Trans. Antennas Propagation, 31, 614

Chu, T. S., Gans, M. J., \& Legg, W. E. 1975, ATT Techn. J., 54, 1665

Goldsmith, P. F. 1992, IEEE Proc., 80, 1729

Kerr, A. R. 1999, IEEE Trans. Microwave Theory Techn., 47, 325

Krebs, N., Barbier, A., Billon-Pierron, D., et al. 2007, IEEE Trans. Appl. Sup., 17,191
Lamb, J. W. 2003, IEEE Trans. Antennas Propagation, 51, 2035

Lopez-Fernandez, I., Gallego, J., Diez, C., \& Barcia, A. 2006, in Microwave Symposium Digest, IEEE MTT-S International, 1907

Maas, S. A. 1993, Microwave mixers (Artech House, Inc.)

Maier, D., Barbier, A., Lazareff, B., \& Schuster, K. 2005a, in Proc. 16th Int. Symp. on Space Terahertz Technology, 428

Maier, D., Devoluy, S., Schicke, M., \& Schuster, K. 2005b, in Proc. 16th Int. Symp. on Space Terahertz Technology, 33

Maier, D., Billon-Pierron, D., Reverdy, J., \& Schicke, M. 2007, in Proc. 18th Int. Symp. on Space Terahertz Technology, 260

Maier, D., Billon-Pierron, D., Reverdy, J., \& Schicke, M. 2008, in Proc. 19th Int. Symp. on Space Terahertz Technology, 93

Navarrini, A., \& Lazareff, B. 2003, in Proc. 14th Int. Symp. on Space Terahertz Technology, 450

Pasternack Enterprises, I. 2009a, Model PE 2054

Pasternack Enterprises, I. 2009b, Model PE 2059

Péron, I. P. P., \& Schuster, K. 2001, IEEE Trans. Appl. Sup., 11, 377

QMC Instruments 2007, Free-standing Wire Grid Polarisers, physics Department, Queen MaryAnd Westfield College, Mile End Road, London El 4NS, UK

Robieux, J. 1959, IEEE Trans. Antennas Propagation, 7, 118

Sanchez Contreras, C., Velilla Prieto, L., Cernicharo, J., et al. 2011, in IAU Symp., 280, 327P

SHI-APD Cryogenics Europe 2007, Sumitomo SRDK-3ST, 3 Hamilton Close, Houndmills Industrial Estate, Basingstoke, Hampshire RG21 6YT, UK

Thomas, B. 1978, IEEE Trans., Antennas Propagation, 26, 367

Virginia Diodes Inc. 2007, Wide band varactor multipliers, 979 Second Street, S.E. Suite 309 Charlottesville, VA

Weiß, A., Ivison, R. J., Downes, D., et al. 2009, ApJ, 705, L45

Wilson, T. L., Rohlfs, K., \& Httemeister, S. 2009, Tools of radio astronomy (Springer) 